\title{
Tumor del estroma gastrointestinal como causa de hemorragia severa; abordaje multidisciplinario. Informe de un caso
}

Palabras clave: Tumor del estroma gástrico, hematemesis, sangrado del tubo digestivo, abordaje multidisciplinario.

Key words: Gastric stromal tumor, hematemesis, gastrointestinal bleeding, multidisciplinary approach.

* Residente de Angiología y Cirugía Vascular, Hospital Regional "Lic. Adolfo López Mateos", ISSSTE. Ciudad de México, México. ** Residente de Anatomía Patológica, Hospital General de Occidente. Zapopan, Jalisco, México. *** Cirujano General. Centro Médico del Instituto de Seguridad Social del Estado de México y Municipios. Toluca, Estado de México, México. **** Residente de Cirugía General. Centro Médico del Instituto de Seguridad Social del Estado de México y Municipios. Toluca, Estado de México,

México.

***** Cirujano

Cardiovascular. Centro Médico del Instituto de Seguridad Social del Estado de México y Municipios. Toluca, Estado de México,

México.

Recibido: 19/02/2016 Aceptado: 02/05/2016

\section{Gastrointestinal stromal tumor as a cause of severe bleeding; multidisciplinary approach. A case report}

Rodrigo Lozano-Corona, ${ }^{*}$ Iris Gabriela Camacho-Partida, ${ }^{* *}$

José Rogelio González-García,*** Salvador Moya-Jiménez,****

Cándido Pedro Chávez-López, ${ }^{* * *}$ M Fernando Rodríguez-Ortega*****

\section{RESUMEN}

Introducción: La hemorragia digestiva baja representa hasta el $2 \%$ de las admisiones hospitalarias por patologías agudas; el colon es responsable del $95 \%$ de estos casos. Cuando el sitio de sangrado difiere, ese 5\% — denominado de origen oscuro - representará un reto diagnóstico destinado a consumir en su búsqueda más tiempo, estudios, atención multidisciplinaria, etcétera. Si la entidad hemorrágica de origen incierto se manifiesta de forma abrupta, sin dar lugar al uso de recursos, se debe obviar el camino conservador y actuar de forma inmediata para erradicar algo desconocido, cuya ubicación es incierta y su actuar agresivo, similar a una bomba de tiempo. Descripción del caso: Presentamos el caso de un paciente portador de un tumor intestinal que dio lugar a episodios de sangrado de origen oscuro y, posteriormente, a una hemorragia digestiva severa.

\section{ABSTRACT}

Introduction: Lower gastrointestinal bleeding accounts for up to $2 \%$ of hospital admissions for acute conditions; the small bowel is responsible for $95 \%$ of these cases. When the bleeding site differs, the $5 \%$ - the so-called bleeding of obscure origin - represents a diagnostic challenge that will require more time, further research and multidisciplinary care. If the gastrointestinal bleeding of unknown origin manifests abruptly, without giving time to the use of any other resources, we should ignore the conservative path and take immediate action to help eradicate something unknown, whose location and behavior are uncertain, similar to a ticking time bomb. Case description: We report this case involving a patient with an intestinal tumor, which initially manifested by bleeding of obscure origin and later progressed into severe gastrointestinal bleeding.

\section{INTRODUCCIÓN}

T os tumores del estroma gastrointestinal _-cuyo acrónimo es GIST, por sus iniciales en inglés (gastrointestinal stromal tumors)— son una entidad patológica relativamente nueva. Los GIST son un subgrupo de sarcomas de tejidos blandos originados en las células intersticiales de Cajal de la pared del intestino. Estos tumores se caracterizan porque expresan el receptor de la tirosin-kinasa CD117.

Los sarcomas de tejidos blandos, en general, son neoplasias que provienen principalmente del mesodermo.
Con la llegada de los métodos inmunohistoquímicos, se ha logrado caracterizar a los tumores GIST por su expresión al receptor de la kinasa de tirosina KIT (CD117). El c-kit es un protooncogén que codifica al KIT, una glicoproteína que funciona como receptor de tipo III transmembranal de la kinasa de tirosina, que una vez activado, produce una cascada de señales intracelulares que regulan la proliferación y diferenciación celular.

La tirosin-kinasa (TK) es un receptor catalítico que se activa por su unión a una molécula señal y acelera la fosforilación del aminoácido tirosina. Esta fosforilación tiene lugar sobre la 
molécula proteica del receptor en el dominio citoplasmático, por lo que se dice que se trata de una autofosforilación.

Los tumores GIST son el tipo más frecuente de tumores mesenquimatosos del tubo digestivo, pero representan únicamente entre el 1 y $3 \%$ de las neoplasias malignas gastrointestinales; el $70-90 \%$ de ellos se encuentra en el estómago y el intestino delgado. ${ }^{1}$

La edad media de diagnóstico es a los 60 años, identificándose un predominio en el sexo masculino. Sólo el $70 \%$ de los pacientes con GIST son sintomáticos. El 20\% son asintomáticos y el $10 \%$ se identifica en autopsias. En años recientes, se asociaron como causa de hemorragia de tubo digestivo de origen desconocido (30$40 \%$, siendo la manifestación más común después del dolor abdominal (60-70\%). El sangrado gastrointestinal bajo se puede originar a nivel de yeyuno, íleon, colon y recto. Estas porciones del tubo digestivo representan una gran extensión de epitelio susceptible de presentar diversas alteraciones, las cuales se pueden manifestar de manera sutil o abrupta, e incluso, representar un riesgo a la vida del individuo; tal es el caso de la hemorragia masiva del tubo digestivo. ${ }^{1-3}$ Cuando la porción afectada es el intestino delgado, nos enfrentamos a un reto diagnóstico y terapéutico, pues la mayoría de las herramientas diagnósticas resultarán insuficientes y las condiciones del sujeto exigen la resolución inmediata del problema: estabilización hemodinámica y remisión del sangrado.

Presentamos el caso de un enfermo masculino joven con antecedente de sangrado de tubo digestivo de origen oscuro, quien durante el protocolo de estudio, acudió al servicio de urgencias con datos de sangrado gastrointestinal bajo masivo. ${ }^{3}$ El objetivo del presente artículo es establecer el contraste entre el protocolo externo y de urgencias aplicado a una persona con sangrado de tubo digestivo oscuro masivo, así como reportar el éxito obtenido mediante el tratamiento quirúrgico, no sólo para remitir el cuadro hemorrágico, sino para erradicar la causa del mismo: un tumor del estroma gastrointestinal.

\section{REPORTE DE CASO}

Paciente masculino de 37 años de edad sin historial de enfermedades cronicodegenerati- vas; como único antecedente de importancia, refiere un internamiento hospitalario por hematoquecia y melena, sin otra sintomatología acompañante. En esa ocasión, acudió al servicio médico de urgencias, donde a la exploración se le encontró ligeramente ansioso, con frecuencia cardiaca de 98 latidos por minuto, presión arterial de 120/70 mmHg; datos compatibles con choque hipovolémico grado I según la clasificación del American College of Surgeons (ACS). Se dio inicio a la reanimación hídrica con soluciones cristaloides.

En la exploración abdominal no se encontraron datos de irritación peritoneal, únicamente dolor a la palpación profunda en la fosa iliaca izquierda. A la exploración rectal se advirtió una tumoración sin datos de sangrado activo.

Se le realizaron estudios de laboratorio, los cuales reportaron leucocitos de 7,000, neutrófilos $58 \%$, plaquetas 260,000 , glucosa $99.8 \mathrm{mg} /$ $\mathrm{dl}$, creatinina $0.84 \mathrm{mg} / \mathrm{dl}$, urea $20.4 \mathrm{~g} / \mathrm{dl}$, tiempo de protrombina 11.5 segundos, INR 1.01, tiempo parcial de tromboplastina 30 segundos; la biometría hemática reportó hemoglobina de $11.1 \mathrm{~g} / \mathrm{dl}$ y hematocrito de $31.9 \%$.

Continuando con el protocolo de estudio, se realizó una endoscopia alta que reportó gastropatía erosiva antral moderada en cicatrización, sin documentarse hemorragia activa ni evidencia de sitio de sangrado. Posteriormente, se sometió a colonoscopia, que produjo como hallazgos únicamente restos hemáticos a nivel del colon e íleon distal. La hematoquecia y la melena remitieron de manera espontánea 24 horas después de su ingreso a urgencias, por lo que el paciente fue egresado, dándosele seguimiento en consulta externa, previa realización de diferentes estudios paraclínicos.

Acudió a valoración con los siguientes resultados: hemoglobina de $15.9 \mathrm{mg} / \mathrm{dl}$, sangre oculta en heces positiva, serie esofagogastroduodenal normal, tránsito intestinal normal, colon por enema con patrón mucoso y haustral sin alteraciones, angiogammagrafía abdominal con eritrocitos marcados negativa para sangrado del tubo digestivo, enteroscopia sin alteraciones y sin restos hemáticos recientes o antiguos; tomografía axial computarizada de abdomen simple y con doble contraste, sin evidencia de imágenes sugerentes de patología tumoral o masa ocupativa intraabdominal. Ante 
tales hallazgos, se solicitó una endoscopia con cápsula entérica. Sin embargo, antes de realizar el estudio, el individuo reingresó al servicio de urgencias con el antecedente de hematoquecia en siete ocasiones, ocurridas en la hora previa a su ingreso, acompañado de diaforesis. En el área de urgencias, se documentó choque hipovolémico grado IV del ACS. Se inició estabilización mediante soluciones cristaloides y transfusión de cuatro concentrados eritrocitarios. Al continuar con datos de choque hipovolémico y hematoquecia, se decidió un tratamiento quirúrgico, con el diagnóstico de sangrado gastrointestinal bajo masivo más choque hipovolémico grado IV.

Se realizó laparotomía exploradora, en la que se encontró una tumoración exofítica dependiente de intestino delgado de aproximadamente $3 \mathrm{~cm}$ de diámetro, macroscópicamente irregular, vascularizada en sus extremos por vasos de mediano calibre, ubicada a $200 \mathrm{~cm}$ de la

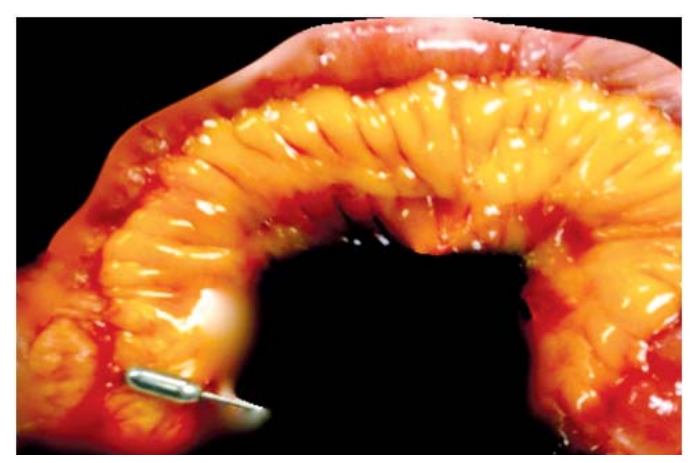

Figura 1. Tumoración exofitica de $3 \mathrm{~cm}$ de diámetro ubicada en la cara antimesentérica del intestino delgado.

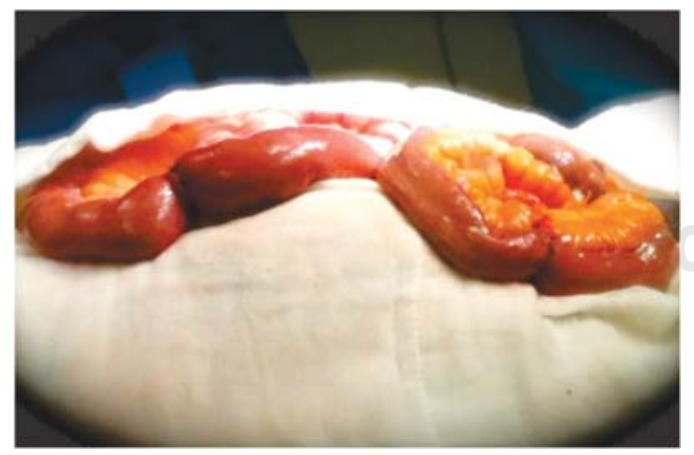

Figura 2. Entero-entero anastomosis intestinal términoterminal en dos planos. válvula ileocecal; se llevó a cabo resección de la tumoración, abarcando un total de $15 \mathrm{~cm}$ de intestino delgado (Figura 1); posteriormente, se restituyó el tránsito intestinal mediante anastomosis término-terminal en dos planos de sutura (Figura 2). El sujeto cursó las primeras 24 horas del postoperatorio en el área de terapia intensiva, sin complicaciones; luego, ingresó a hospitalización de cirugía general. Durante su estancia permaneció estable y sin presentar datos de hemorragia digestiva, tolerando adecuadamente la dieta normal; el enfermo fue egresado por mejoría.

El reporte por patología macroscópica de la resección de intestino delgado muestra una lesión nodular submucosa de $2.5 \mathrm{~cm}$ de diámetro mayor, bordes bien definidos, blanquecina, de aspecto arremolinado y consistencia firme (Figura 3). El diagnóstico microscópico fue de un tumor del estroma gastrointestinal de bajo potencial maligno, corroborado por inmunohistoquímica (Figuras 4 y 5). A ocho meses de seguimiento postoperatorio, el paciente no refiere nuevos episodios de hematoquecia o melena; los estudios de laboratorio de control muestran la hemoglobina dentro de parámetros normales y el estudio de sangre oculta en heces es negativo.

\section{DISCUSIÓN}

La hemorragia digestiva baja es una entidad frecuente que representa del 1 al $2 \%$ de las ad-

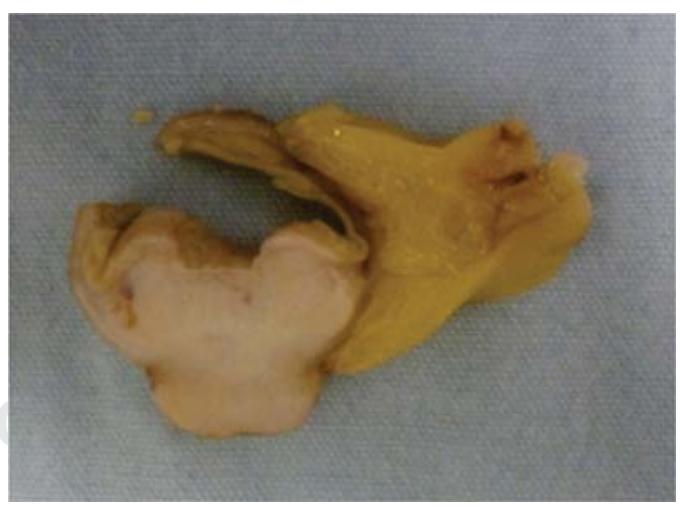

Figura 3. Pieza de patología. Evaluación macroscópica de intestino delgado corte coronal, donde se aprecia una lesión nodular submucosa de $2.5 \mathrm{~cm}$ de diámetro mayor, bordes bien definidos, blanquecina, aspecto arremolinado y consistencia firme. 


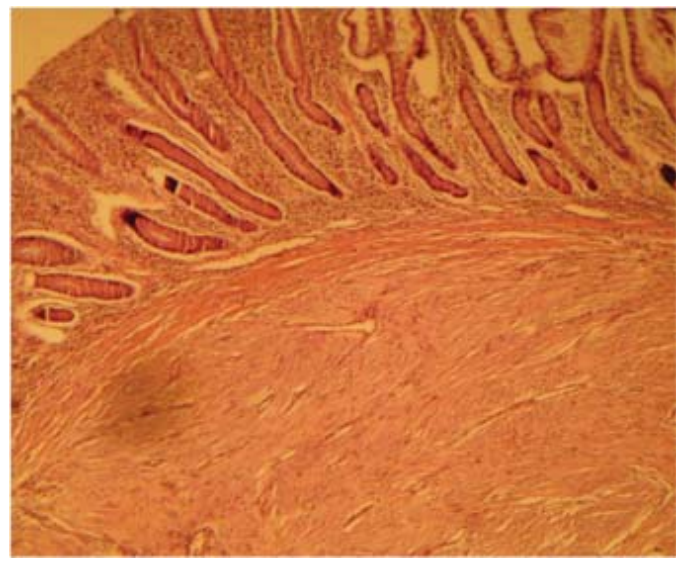

Figura 4. Aspecto microscópico con tinción de hematoxilinaleosina. Lesión fusocelular mesenquimatosa de localización submucosa.

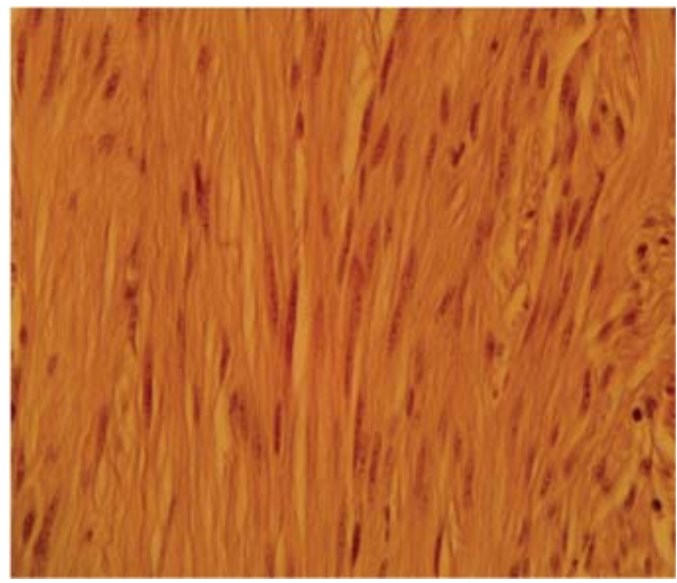

Figura 5. Aspecto microscópico con tinción de hematoxilinaleosina. Se observan haces de células fusiformes, de núcleos ovoides, con cromatina granular fina. No se identificaron mitosis atípicas, necrosis ni pleomorfismo celular.

misiones hospitalarias por patologías agudas. El colon es el responsable hasta del $95 \%$ de estos casos; sin embargo, el 5\% restante representa generalmente un reto diagnóstico. ${ }^{1,2}$ El sangrado de tubo digestivo es la segunda causa de transfusión masiva (definida como la administración de cuatro o más concentrados eritrocitarios en una hora) en padecimientos de urgencia, después del politrauma., ${ }^{3,4} \mathrm{Di}$ cha hemorragia se considera masiva cuando requiere más de dos a seis unidades de sangre en 24 horas, en el contexto de un perfil de coagulación dentro de parámetros normales. De 10 a $45 \%$ de estos individuos requerirán cirugía urgente; la tasa de mortalidad se eleva hasta un $22 \% .{ }^{4} \mathrm{El}$ sangrado digestivo severo o masivo se manifiesta en su mayoría mediante melena $(75 \%)$, seguida de hematemesis $(50 \%)$ $y$, con menor frecuencia, la presencia de hematoquecia $(14 \%){ }^{3}$ ocurre principalmente en sujetos de edad avanzada y patologías agregadas. Sin embargo, puede aparecer a cualquier edad y en individuos sin antecedentes previos de enfermedad, ${ }^{4}$ tal es el caso de la persona que presentamos.

En adultos jóvenes y adolescentes, la principal etiología de sangrado es el divertículo de Meckel, la enfermedad intestinal inflamatoria y los pólipos intestinales. En adultos mayores de 60 años - e incluso menores-, las causas más comunes de hemorragia digestiva son divertículos colónicos, angiodisplasias, neoplasias y la enfermedad intestinal inflamatoria. La enfermedad diverticular representa la principal causa de sangrado de manera global. Se origina de la erosión de un divertículo que emerge adyacente a un vaso sanguíneo y se resuelve de forma espontánea en el 70 a $80 \%$ de los casos, con un porcentaje de resangrado de 25 a $30 \%$ y riesgo del $50 \%$ de un tercer episodio de hemorragia. ${ }^{1}$ Las angiodisplasias incluyen a las ectasias vasculares, malformaciones arteriovenosas o hemangiomas; son la causa más frecuente de sangrado en pacientes de edad avanzada. La remisión espontánea ocurre en el $90 \%$ de los casos, la tasa de resangrado es similar a la enfermedad diverticular y sólo presentan hemorragia masiva $15 \%$ de los individuos. ${ }^{1}$ Las ectasias vasculares son anormalidades a nivel de vasos sanguíneos caracterizadas por acumulación focal y dilatación de venas en la mucosa y submucosa intestinal, no asociadas con lesiones angiomatosas de la piel u otras vísceras. La causa probable es una obstrucción de bajo grado, parcial o intermitente de venas submucosas en el sitio donde los vasos traspasan las capas musculares del colon. Por último, los anillos capilares se dilatan y se produce una pequeña fístula arteriovenosa. ${ }^{5}$ Las angiodisplasias del intestino delgado representan el $16 \%$ del total de dicha entidad a nivel del tracto digestivo. Se 
pueden presentar a cualquier edad, asociadas a un proceso congénito en menores de 20 años y derivadas de un proceso degenerativo en sujetos mayores de 60 años. ${ }^{6}$ La hemorragia por enfermedad inflamatoria intestinal es poco común; tiene una incidencia del 0.9 al $6 \%$. Las neoplasias que con mayor frecuencia se manifiestan por sangrado son las colorrectales, los eventos hemorrágicos masivos son escasos, representan únicamente el 15\% del sangrado masivo bajo en población adulta. ${ }^{1}$

La hemorragia digestiva de origen oscuro se define como el sangrado persistente o recurrente cuyo origen es imposible de precisar mediante endoscopia alta, colonoscopia y evaluación radiológica. Corresponde al 5\% de las hemorragias gastrointestinales y $50 \%$ presentan episodios recurrentes de hemorragia digestiva. ${ }^{7,8}$ Las lesiones inadvertidas de esófago, estómago (úlceras de Cameron, várices fúndicas, úlcera péptica, angioectasias, lesión de Dieulafoy, ectasia vascular antral, etcétera) y colon en estudios de imagen iniciales, así como las lesiones del intestino delgado, representan en su mayoría la etiología del sangrado denominado oscuro. Causas menos comunes incluyen hemobilia, hemosuccus pancreaticus y fístula aortoentérica. ${ }^{7}$

El abordaje de este tipo de casos inicia con una historia clínica minuciosa, exploración general enfatizada a la región abdominal y rectal armada, monitorización continua invasiva y no invasiva, así como estudios paraclínicos tales como biometría hemática, electrolitos séricos, perfil de coagulación y gases arteriales. Para ayudar a delimitar la localización del sangrado, se debe colocar una sonda nasogástrica, identificando las características del gasto. En cuanto al orden de los estudios a solicitar, muchos grupos recomiendan llevar a cabo colonoscopia de manera temprana. ${ }^{9,10}$ Desde 1974, el uso de la colonoscopia previa preparación intestinal es un instrumento útil para esclarecer el origen de la hemorragia digestiva baja de forma inicial cuando el sangrado ha cedido o disminuido, con ventajas en cuanto a disminución de días de estancia intrahospitalaria cuando se realiza en las primeras 24 horas del ingreso. ${ }^{1}$ Estudios de imagen como la colonoscopia y la cápsula entérica han demostrado disminución en los procedimientos quirúrgicos electivos secun- darios a sangrado digestivo, no así en cirugía de urgencia. ${ }^{10}$

En personas sin anemia, con sangre oculta en heces positiva, estudios más allá de la colonoscopia no han presentado mayor beneficio. En enfermos con anemia ferropénica y estudio de sangre oculta en heces positivo que no presentan sangrado activo, se recomienda repetir los estudios de imagen convencionales; en caso de no identificar el sangrado en los estudios anteriores, se debe realizar enteroscopia con cápsula endoscópica.

La causa más frecuente de este tipo de sangrado son las angiodisplasias (80\%). Sin embargo, en pacientes menores de 50 años, la etiología más común son las neoplasias. En individuos que, además, persisten con melena o hematoquecia, se realiza el protocolo mencionado más toma de biopsias al azar, uso de naloxona inyectada para evidenciar angioectasias oscuras, endoscopio con visión lateral, inyección de glucagón para relajar la ámpula de Vater y reparar aneurismas en personas portadoras. ${ }^{7}$ En sujetos con sangrado activo, se recomienda el uso de cápsula endoscópica para determinar el sitio hemorrágico o descartar al intestino delgado como trayecto afectado, y así, agilizar la búsqueda de hemorragia en colon y tracto digestivo alto. Además, puede reducir los costos del protocolo externo en sangrado oscuro gastrointestinal, ya que es un método relativamente reciente cuya capacidad radica en detectar anomalías intraluminales e intramulares a lo largo de todo el intestino. Sólo el $5 \%$ de las personas con hallazgos negativos mediante dicho método de estudio presentarán nuevamente hemorragia digestiva. Cuando las imágenes por cápsula endoscópica sugieren tumoración, el enfermo debe ser sometido a laparoscopia diagnóstica.

En el caso del paciente presentado, no fue posible realizar la enteroscopia por cápsula debido al cuadro agudo; a la fecha, no es una herramienta disponible en todos los centros hospitalarios. Por la etiología del sangrado, la posibilidad de hallazgos positivos mediante enteroscopia por cápsula pudieron ser altos, e incluso, un auxiliar para ofrecer un procedimiento quirúrgico electivo. Su uso cada vez es más difundido y puede ser un método de imagen importante en la detección de tumores 
de yeyuno e íleon. ${ }^{11}$ Por otro lado, la realización de una tomografía axial computarizada ayuda a llevar a cabo una punción por vía percutánea en individuos con sospecha de GIST, lo que proporciona muestras de tejido fresco y congelado para analizar su morfología y hacer inmunohistoquímica, con la intención de confirmar positividad para receptor de la tirosin-kinasa CD117, ya que con esto no solamente orienta a realizar tratamiento quirúrgico, sino también nos aporta datos encaminados al mal pronóstico, como son localización en íleon distal o colon, tamaño mayor a cinco centímetros, índice mitótico de dos o más mitosis por 50 campos de gran aumento, antígeno de proliferación nuclear mayor al 10\%, infiltración a órganos vecinos y alta aneuploidia. ${ }^{3}$

Los tumores de intestino delgado son poco frecuentes: representan del 3 al 5\% de las tumoraciones del tracto digestivo; la sintomatología más frecuente es el dolor abdominal vago (50 a $75 \%$ ) y la hemorragia digestiva (20 a $50 \%)^{7}$ Se han descrito 40 tipos histológicos diferentes de tumoraciones del intestino delgado; las neoplasias benignas representan del 30 al 50\% de estos tumores; destacan los adenomas, lipomas, hamartomas y hemangiomas. Del cáncer, de 35 a 50\% corresponde a adenocarcinomas, de 20 a $40 \%$ a tumores neuroendocrinos, y los linfomas comprenden el $1 \%$. Los tumores del estroma gastrointestinal (GIST) abarcan únicamente el 15\%. Las localizaciones más frecuentes son el íleon (30\%) y el duodeno (25\%). El 50\% de las neoplasias de intestino delgado son asintomáticas; su presentación más común es la obstrucción parcial del tránsito (25\%); otro $25 \%$ presenta masa abdominal palpable. Los GIST tienen mayor predisposición a acompañarse de hemorragia manifiesta. ${ }^{12,13}$

La cirugía sigue siendo el pilar del tratamiento para los sujetos con GIST primario sin evidencia de metástasis, teniendo en cuenta que es la terapia inicial si el tumor es técnicamente resecable. En este caso, la decisión de realizar laparotomía exploradora fue aceptable, ya que se valoraron a fondo las superficies peritoneales y órganos adyacentes con la intención de verificar la existencia de metástasis, mantener la integridad de la cápsula del tumor, y evitar sangrado y diseminación celular. Las dimensiones macroscópicas ayudaron a determinar la decisión de resección del tumor, ya que era de $3 \mathrm{~cm}$ (el criterio de resecabilidad en la literatura es mayor de $2.5 \mathrm{~cm}$ ); de igual forma, la resección intestinal mayor de $10 \mathrm{~cm}$ está indicada para verificar márgenes microscópicos negativos. ${ }^{14}$

En este caso, no se realizó linfadenectomía, ya que es innecesaria debido a que la metástasis en los ganglios linfáticos es poco frecuente con GIST y sarcomas en general. ${ }^{14}$

La evaluación trans- y postoperatoria por el servicio de patología es esencial para confirmar el diagnóstico después de la intervención quirúrgica. El 90\% de los GIST esconden mutaciones que activan el c-KIT, favoreciendo la proliferación celular, y deprimen la apoptosis en el tumor; aquí la importancia de la vigilancia estrecha por parte de imagenología realizando tomografía axial computarizada pélvica cada seis meses, ya que es la prueba de elección para el seguimiento. Oncología médica y oncología quirúrgica son los responsables directos de la vigilancia postoperatoria, ya que durante el primer año postoperado se tendrá que vigilar cada tres meses por ambos servicios, teniendo el respaldo de los servicios antes mencionados; en caso de existir recidiva del tumor, habrá que valorar el tratamiento médico, como es el imatinib, que es un inhibidor del c-KIT; estudios prospectivos han mostrado que $50 \%$ de las personas responden a este tipo de tratamiento y $80 \%$ mantienen la enfermedad estable. ${ }^{14-16}$

\section{CONCLUSIÓN}

Se deben considerar las tumoraciones de intestino delgado como causa de hemorragia intestinal baja masiva en aquellos pacientes en apariencia sanos y que no se encuentran en los grupos etarios extremos de la vida, en quienes puede manifestarse como una urgencia grave por el estado de choque, como en este caso, donde representó una urgencia absoluta sin un diagnóstico definido.

\section{REFERENCIAS}

1. Azcoitia MF. Reporte de 44 casos CD117 positivos de tumores del estroma gastrointestinal. Un programa mexicano de detección de nuevos casos. Cir Gen. 2008; 30: 127-135. 
2. Rammohan A, Sathyanesan J, Rajendran K, Pitchaimuthu A, Perumal SK, Srinivasan U. A gist of gastrointestinal stromal tumors: a review. World J Gastrointest Oncol. 2013; 5: 102-112.

3. Casali PG, Blay JY, Bertuzzi, Bielack S, Bjerkehagen B, Bonvalot $\mathrm{S}$, et al. Gastrointestinal stromal tumours: ESMO clinical practice guidelines for diagnosis, treatment and follow-up. Ann Oncol. 2014; 25: 21-26.

4. Hoedema ER, Luchtefield AM. The management of lower gastrointestinal hemorrhage. Dis Colon Rectum. 2005; 48: 2010-2024.

5. Schuster KM, Davis KA, Rosernbaum ST. Emergency and urgent surgery. Med Clin North Am. 2009; 93: 1131-1148.

6. Bannura CG, Barrera EA, Melo LC, Contreras PJ, Soto CD. Hemorragia digestiva baja masiva: resultados del estudio y el tratamiento quirúrgico en 20 pacientes consecutivos. Rev Med Chile. 2002; 130: 869-878.

7. Arteaga FM, Blancas VJ. Angiodisplasias del tracto gastrointestinal bajo, diagnóstico y tratamiento. Rev Fac Med UNAM. 2003; 46: 197-201.

8. Niño SJ, Girón QG, Sánchez FP, Blanco BR, Gómez JL, Muñoz OR. Hemorragia del tubo digestivo secundaria a angiodisplasia yeyunal asociada a divertículos yeyunales. Rev Gastroenterol Mex. 2000; 65: 81-84.

9. Raju GS, Gerson L, Das A, Lewis B. American Gastroenterological Association (AGA) Institute technical review on obscure gastrointestinal bleeding. Gastroenterology. 2007; 133: 1694-1696.

10. Barrera EA, Morató AP, Bannura CG, Peñaloza MP, Martínez OD, Massoli ID. Hemorragia digestiva oculta por tumor del intestino delgado. Rev Chil Cir. 2003; 55: 505-508.

11. Lhewa DY, Strate LL. Pros and cons of colonoscopy in management of acute lower gastrointestinal bleeding. World J Gastroenterol. 2012; 21: 1185-1190.
12. Gayer C, Chino A, Lucas C, Tokioka S, Yamasaki T, Edelman DA, et al. Acute lower gastrointestinal bleeding in 1,112 patients admitted to an urban emergency medical center. Surgery. 2009; 146: 600-607.

13. Pennazio M, Rondonotti E, Franchis RD. Capsule endoscopy in neoplastic diseases. World J Gastroenterol. 2008; 14: 5245-5253.

14. Tavakkolizadeh A, Whang EE, Ashley WS, Zinner JM. Small intestine. In: Schwartz's (coord). Principles of surgery. United States of America: McGraw-Hill; 2009. p. 1017-1054.

15. Chapa AO, Ortiz HV, Echegaray DA, Cruz MR. Tumores del estroma gastrointestinal (GIST) ampulares tratados con pancreaticoduodenectomía. Reporte de dos casos y revisión de la literatura. Rev Med Hosp Gen Mex. 2013; 76: 52-56.

16. Demetri GD, von Mehren M, Antonescu CR, DeMatteo RP, Ganjoo KN, Maki RG, et al. NCCN Task Force report: update on the management of patients with gastrointestinal stromal tumors. J Natl Compr Canc Netw. 2010; 41: 42-44.

Correspondencia:

Dr. Rodrigo Lozano Corona

Hospital Regional "Lic. Adolfo López Mateos", ISSSTE.

Departamento de Angiología y Cirugía Vascular.

Av. Universidad Núm. 1321,

Álvaro Obregón, Axotla, 01030,

Ciudad de México, México.

Teléfono: 0445510531541

E-mail: rodrigo_lc77@me.com 